\title{
Structural Characterization and Electrical Property of the Manganese Oxides/Silver Nanocomposite Thin Films
}

\author{
Yi Hu, Jiun-Shing Liu, and Tung-Cheng Liu
}

\begin{abstract}
The microstructural characterization and electrical properties of the manganese oxides/silver nanocomposite thin films are investigated by the conducting probe atomic force (CP-AFM). The nanocomposite thin films were obtained thriugh electrodeposited by potentiostatic method with silver acetate $\left(\mathrm{AgC}_{2} \mathrm{H}_{5} \mathrm{OOH}\right)$ and potassium permanganate $\left(\mathrm{KMnO}_{4}\right)$ aqueous solution. The morphology for the thin films was examined by atomic force microscopy (AFM), Scanning Electronic Microscopy (SEM) and transmission electron microscopy (TEM). The spherical $\mathrm{Ag}_{2} \mathrm{O}$ nanoparticles of several nanometer are dispersed homogeneouly in the thin films and cubic shape Ag nanoparticles of about about $100 \mathrm{~nm}$ are spreaded on the surface of $\mathrm{MnOx}$ thin film. The thickness of the MnOx thin film is about $250 \mathrm{~nm}$. The schottky junction between $\mathrm{Ag}$ and $\mathrm{MnOx}$ with rectifying behavior was evidenced by CP-AFM measurement. The turn-on voltage for the junction is around $0.69 \mathrm{~V}$.
\end{abstract}

Index Terms-Silver nanoparticles, manganese oxides, thin film, electrodeposition.

\section{INTRODUCTION}

Manganese oxides are widely used for energy storage devices due to low cost, high energy density, environment pollution free and nature abundance [1]-[3]. A lot of types of manganese oxides have been investigated, including nanocrystalline and amorphous phases [4]-[6]. In these oxides, $\mathrm{MnO}_{2}$ thin films for the supercapactive electrode exhibited a relative high specific capacitance (SC) of $700 \mathrm{~F} / \mathrm{g}$ as reported [7], [8]. However, the $\mathrm{SC}$ of the $\mathrm{MnO}_{2}$ electrode decreased with the increase of film thickness due to the poor conductivity of $\mathrm{MnO}_{2}\left(10^{-5}-10^{-6} \mathrm{Scm}^{-1}\right)$ and limits the charge/discharge rate for high-power applications [9]. On the other hand, non-stoichiometric manganese hydroxide $\mathrm{MnO}_{\mathrm{x}}$ also has good SC properties, and can be obtained by converting the manganese hydroxide $\left(\mathrm{Mn}(\mathrm{OH})_{2}\right)$ [10], [11]. The $\mathrm{Mn}(\mathrm{OH})_{2}$ films can be fabricated by cathodic electrodeposition from $\mathrm{Mn}^{2+}$ salts solution.

Such a nanostructured film usually gives much higher energy storage density. The performance of the energy devices are basically determined by the structural and electrochemical properties of electrode materials. Nevertheless, the electrical capacity of supercapacitor cell depends on the electron transport mechanism and the type of series connection resistance of the cell. The resistance of the

Manuscript received January 5, 2013; revised March 4, 2013. This work was supported by National Science Council, Taiwan, ROC, under the grant NSC 99-2221-E-036-013.

The authors are with Department of Materials Engineering, Tatung University, Taipei, Taiwan, ROz (e-mail: huyi@ttu..edu.tw, scottsym@yahoo.com.tw, emptylau@hotmail.com). electrode must be decreased to a proper value to give high electronic transport and low charge leakage.

In this study, the manganese oxides thin films were electrodeposited on the and ITO glass substrates with potassium permanganate solution by potentiostatic method. In addition, sliver was added as the conduction drug to improve the conducting properties of the manganese oxides thin film. The potassium permanganate was adopted in this work is because that it is an oxidizing agent widely used as reported [12]. This would enhance to co-deposite Ag with the manganese oxides at the same side.

\section{EXPERIMENTAL DETAILS}

The electrochemical deposition and characterization of the thin films were conducted at room temperature using a Jichan 5000 electrochemical workstation in a standard cell with a platinum counter electrode, and a SCE or $\mathrm{Ag} / \mathrm{AgCl}$ reference electrode. The thin films were electrochemical deposited on ITO glass substrate with potentiostatic method $(-0.7 \mathrm{~V}$ vs. open circuit) for $200 \mathrm{~s}$.

The solutions for the deposition are prepared with the potassium permanganate and silver acetate dissolved in deionized water. The concentration of potassium permanganate is $0.1 \mathrm{M}$ and the molar ratio of the $\mathrm{Ag} / \mathrm{Mn}$ in the solution is $1.6 \%$.

The morphology of the thin film was studied using a field emission scanning electronic microscope (FESEM, Hitachi 4800). The states of the ions were analyzed using an X-ray photoelectron spectroscopy (XPS, VG ESCA Scientific Theta Probe) under $\mathrm{Al} \mathrm{K} \alpha(1486.6 \mathrm{eV})$ and the X-ray spot size is $15 \mu \mathrm{m}$. The surface morphology and the surface electrical properties were conducted by the AFM (Digital Instrument NS3a controller with D3100). Commercial sharpened PtIr ${ }_{5}$ coating tips attached to triangular cantilevers were used. The set point was adjusted to minimize the force between the tip and the sample in each measurement.

The I-V characteristic of the sample was also measured by Conductive Atomic Force Microscopy (CP-AFM). CP-AFM was used to examine the surface morphology and electrical properties distributed of the films. Atomic force microscopy (AFM) is normally used to study the topography of substrates and structures made on them. Scanning of the sample is done with a sharp tip on a cantilever. If the cantilever and tip are made from of electrically conducting material, electrical properties of the films can be studied at the same time.

\section{RESULTS AND DISCUSSION}

Fig. 1 shows the SEM surface micrograph of the 
manganese oxides thin films. It was found that cubic shape particles and near-spherical smaller particles precipitated on the surface of the thin films. The size of the near-spherical particles was about $30 \sim 50 \mathrm{~nm}$ and that of the cubic shape particles was about $100 \mathrm{~nm}$. The thickness of the $\mathrm{MnOx}$ thin film is about $250 \mathrm{~nm}$. The composition of the particles on the thin films was identified as the $\mathrm{Ag}$ from the EDX investigation. (the left top in Fig. 1) The EDX spectrum of the sample shows the presence of $\mathrm{Mn}, \mathrm{Ag}$ and $\mathrm{O}$ as the major elements at the vicinity of the cubic particles. In addition, peaks corresponding to Si and In form ITO glass substrates are also present. Therefore, the main composition of the cubic particles is Ag from EDX analysis.

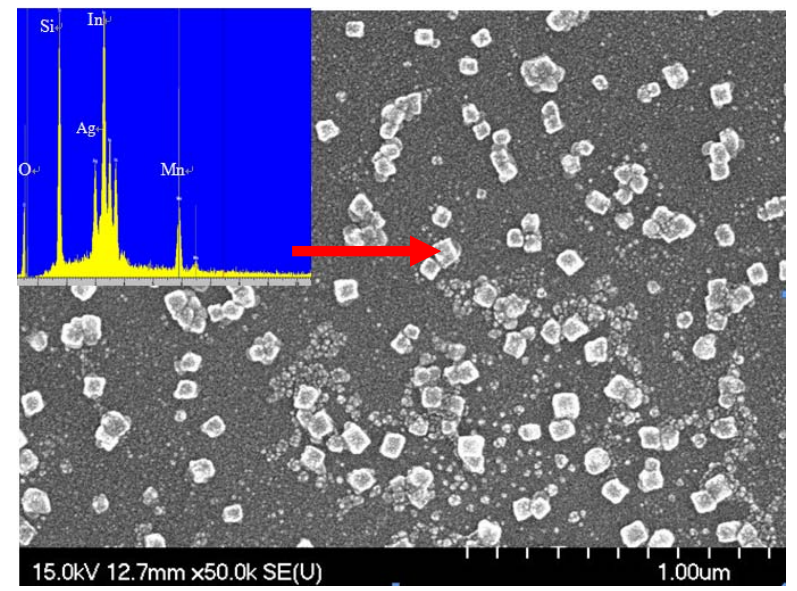

Fig. 1. The SEM micrographs of the thin films and the EDX spectrum of the particles (as arrowed)

Fig. 2 shows the TEM micrographs for the $\mathrm{MnO}_{\mathrm{x}}$ matrix of the thin film. It was found that the $\mathrm{MnO}_{\mathrm{x}}$ matrix has the near-spherical nanoparticles embedded into the films as in Fig. 2 (a). The size of the nanoparticles (darker particles) varied from several nanometer to $30 \mathrm{~nm}$. The typical diffraction pattern of spherical nanoparticles was shown in Fig. 2 (b). The diffraction pattern of the nanoparticles was classified as the single phase of $\mathrm{Ag}_{2} \mathrm{O}$ with primitive $\mathrm{FCC}$ structure (JCPDF: 76-1393). On the other hand, the diffuse ringing diffraction pattern of the matrix indicates that the matrix $\mathrm{MnO}_{\mathrm{x}}$ thin film is near amorphous as in Fig. 2 (c). This is well agreement to that the thin film is composed of the $\mathrm{MnO}_{\mathrm{x}}$ nanoparticles of about $10 \mathrm{~nm}$ form SEM observation.

On the other hand, Fig. 3 shows the TEM micrographs of the cubic particles, which dispersed on the surface of the $\mathrm{MnO}_{\mathrm{x}}$ thin films. The main composition of the cubic shape particle is identified as metal Ag from the EDX spectra analysis as in Fig. 3. The spotty diffraction pattern suggests the nanoparticles of silver metal with FCC structure. This is well agreement to the result of SEM analysis. Therefore, the microstructure of the thin films is that the spherical $\mathrm{Ag}_{2} \mathrm{O}$ nanoparticles of several nanometer are dispersed homogeneouly in the thin films and cubic shape $\mathrm{Ag}$ nanoparticles of about about $100 \mathrm{~nm}$ spreading on the surface of $\mathrm{MnO}_{\mathrm{x}}$ thin film.

Fig. 4 shows the XPS spectrum of Mn2p3/2 of the thin film. The binding energy of the Mn2p3/2 for the manganese oxidation state is $640.9 \mathrm{eV}$ for the $\mathrm{Mn}^{2+}, 641.9 \mathrm{eV}$ for the $\mathrm{Mn}^{3+}, 642.5 \mathrm{eV}$ for the $\mathrm{Mn}^{4+}$ as reported in literature [13]. In our study, Therefore, the predominant phase of the thin film is $\mathrm{MnO}_{2}$ with small amount of $\mathrm{Mn}_{2} \mathrm{O}_{3}$. The manganese oxidation state of this film was $17.8 \mathrm{at} \%$ for $\mathrm{Mn}^{3+}$ and 82.2 at $\%$ for $\mathrm{Mn}^{4+}$ from evolution of the spectra.

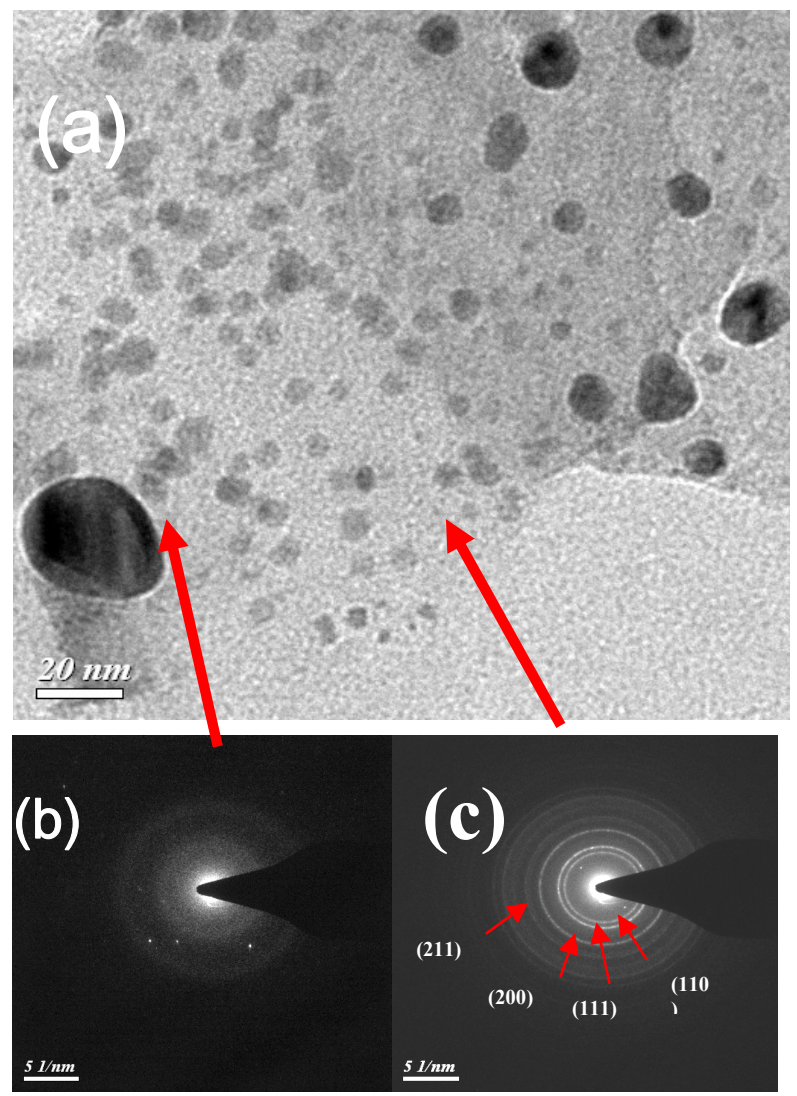

Fig. 2. TEM micrographs and electron diffraction patterns of the darker nanoparticles and $\mathrm{MnO}_{\mathrm{x}}$ thin film matrix. The darker nanoparticles are identified as $\mathrm{Ag}_{2} \mathrm{O}$ crystallites.

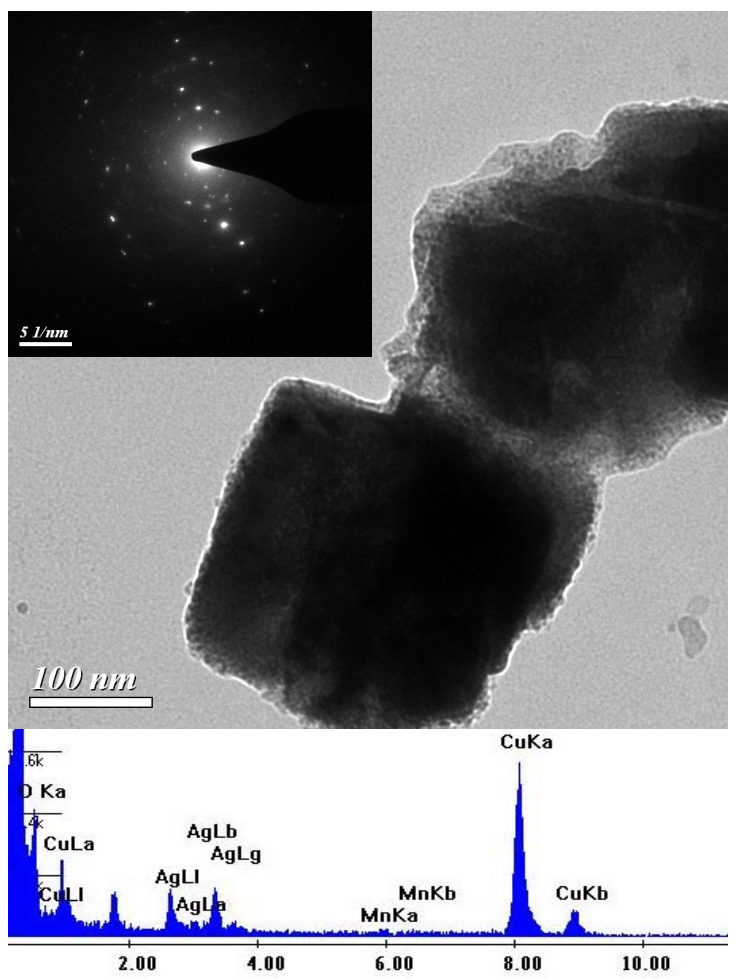

Fig. 3. TEM micrographs and electron diffraction pattern of the cubic shape particles

Fig. 5 shows the surface morphology and the surface electrical conducting imagines of the thin films, the with 
lighter particles as observed in Fig (5.a) are Ag nanoparticles. The size of the particles is about $200 \mathrm{~nm}$ measurement from AFM. This is very close to the observation from FESEM analysis.

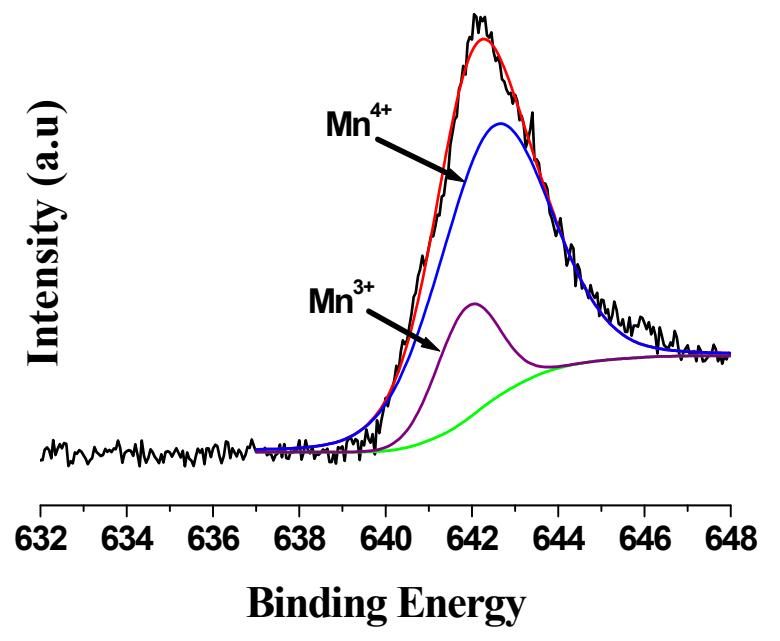

Fig. 4. The XPS spectra of Mn2p3/2 of the sample. This peak was divided into two spectra with $\mathrm{Mn}^{3+}$ and $\mathrm{Mn}^{4+}$.
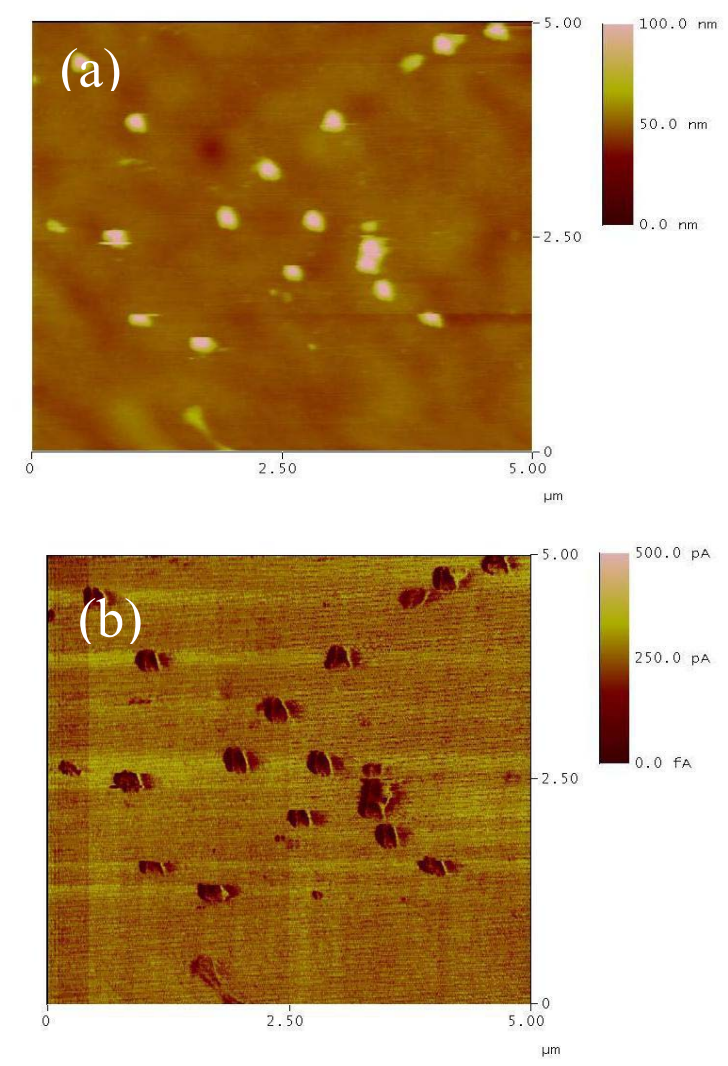

Fig. 5. Surface morphologies of the thin films scanned by the CP-AFM. (a) in normal mode, and (b) in conduting mode.

On the other hand, these Ag particles are darker spots from CP-AFM scanning as in Fig. 5(b). This is ascribed to the lower current through to Ag nanoparticles. The lower current through the $\mathrm{Ag}$ nanoparticles is primarily due to the metal-semiconductor contacts as Schottky barriers. A metal-semiconductor junction that does rectify current is called an schottky contact. It has been reported that a saddle potential shape underneath the nano-Schottky contacts was considerably modified by the surface Fermi level pinning on the air exposed free surfaces [14].

This suggested that the potential underneath the Schottky contact between the $\mathrm{MnO}_{\mathrm{x}}$ matrix and silver particle is higher than that in the interior of the silver nanoparticles and $\mathrm{MnO}_{2}$ matrix. The electrons in the silver nanoparticle with lower potential should overcome the Schottky barrier to transport. However, silver nanoparticles would become insulating if the applied field is lower than the Schottky barrier as seen in Fig. 5 (b).

Fig. 6 shows the structure, current-voltage performance, of our device. It shows a typical forward $I-V$ characteristic of an $\mathrm{Ag}$ and $\mathrm{MnOx}$ junction measured at $300 \mathrm{~K}$. This I-V characteristic shows the typical shottky barrier behavior and confirms the explanation of the darker spots of $\mathrm{Ag}$ nanoparticles in Fig. 5 (b). The resistance of the Schottky barrier in the field emission regime is quite low as shown from the step increase in current at about $0.06 \mathrm{~V}$. in the It was measured by CP-AFM bias voltage with a step of $0.06 \mathrm{~V}$. The turn-on voltage for the sample, therefore, is around $0.69 \mathrm{~V}$ from CP-AFM measurement. This Schottky behavior have a much faster response under forward bias conditions. This may be attributed to the highly conducting behavior of the $\mathrm{MnOx}$ thin films and thus gives a low series resistance of the junction.

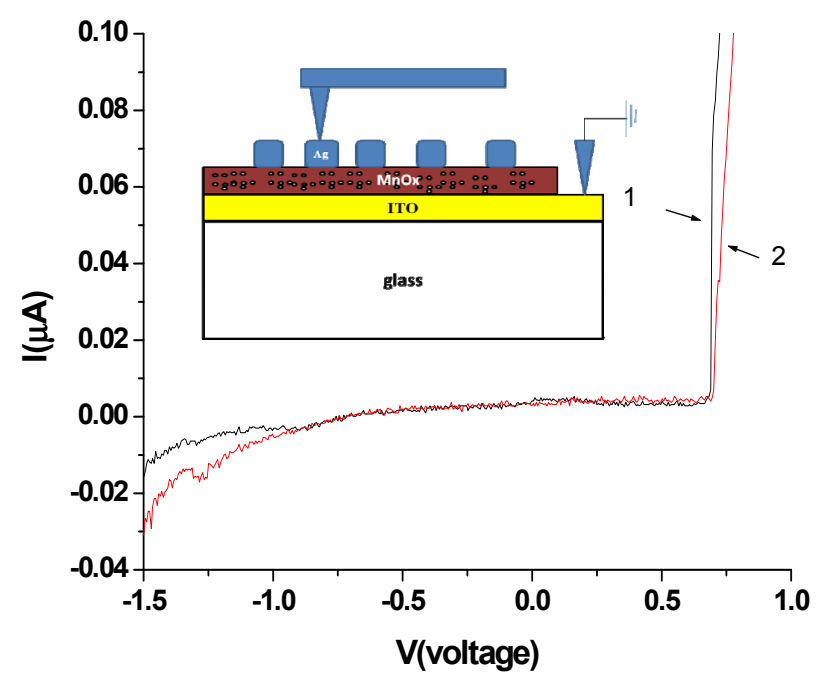

Fig. 6. The I-V characteristics of the thin films measured by CP-FAM. The scan of the I-V measurement was repeated twice. The number shown in figure indicates the first time and second time scanning.

\section{CONCLUSION}

Manganese oxides/silver nanocomposite thin films were prepared by electrodeposited on ITO substrate. Silver particles of about $100 \mathrm{~nm}$ spreaded on the surface of the $\mathrm{MnO}_{\mathrm{x}}$ thin film, whereas the $\mathrm{Ag}_{2} \mathrm{O}$ nanoparticles precipitated in the thin films. The thickness of the $\mathrm{MnOx}$ thin film is about $25 \mathrm{~nm}$. Schottky barrier was obtained from the variation of electrical conducting behavior between the silver nanoparticles and $\mathrm{MnO}_{2}$ martix. The turn-on voltage for forward bias was obtained around $0.69 \mathrm{~V}$.

\section{REFERENCES}

[1] S. Komaba, A. Ogata, and T. Tsuchikawa, "Enhanced supercapacitive behaviors of birnessite," Electrochem Commun., vol. 10, pp.1435-1437, 2008.

[2] R. N. Reddy and R. G. Reddy, "Sol-gel $\mathrm{MnO}_{2}$ as an electrode material for electrochemical capacitors," J. Power Sources, vol. 124, pp. 330-335, 2003. 
[3] M. Toupin, T. Brousse, and D. Belanger, "Charge storage mechanism of $\mathrm{MnO}_{2}$ electrode used in aqueous electrochemical capacitor," Chem. Mater., vol. 16, pp. 3184, 2004.

[4] C. C. Hu and T. W. Tsou, "Ideal capacitive behavior of hydrous manganese oxide prepared by anodic deposition," Electrochem. Commun., vol. 4, pp. 105, 2002.

[5] E. Beaudrouet, A. Le Gal La Salle, and D. Guyomard, "Nanostructured manganese dioxides: Synthesis and properties as supercapacitor electrode materials," Electroch. Acta, vol. 54, pp. 1240, 2009.

[6] M. S. Wu and P. C. J. Chiang, "Fabrication of nanostructured manganese oxide electrodes for electrochemical capacitors," Electrochem. Solid-State Lett., vol. 7, pp. 123, 2004

[7] S. C. Pang, M. A. Anderson, and T. W. Chapman, "Novel electrode materials for thin-film ultracapacitors: Comparison of electrochemical properties of sol-gel-derived and electrodeposited manganese dioxide," J. Electrochem. Soc., vol. 147, pp. 444, 2000.

[8] S. C. Pang and M. A. Anderson, "Novel electrode materials for electrochemical capacitors: Part II. Material characterization of sol-gel-derived and electrodeposited manganese dioxide thin films," $J$. of Mater. Res., vol. 15, pp. 2096, 2000.

[9] X. Lang, A. Hirata, T. Fujita, and M. Chen, "Nanoporous metal/oxide hybrid electrodes for electrochemical supercapacitors," Nature Nanotech. vol. 6, pp. 232-236, 2011.

[10] N. Nagarajan, H. Humadi, and I. Zhitomirsky, "Cathodic electrodeposition of $\mathrm{MnOx}$ films for electrochemical supercapacitors," Electrochim. Acta., vol. 5, pp. 3039, 2006.

[11] N. Nagarajan, M. Cheong, and I. Zhitomirsky, "Electrochemical capacitance of MnOx films," Mater. Chem. Phys., vol. 103, pp. 1, 2007.

[12] D. Zhao, Z. Yang, L. Zhang, X. Feng, and Y. Zhang, "Electrodeposited manganese oxide on nickel foam-supported carbon nanotubes for electrode of supercapacitors," Electrochem.l and Solid-State Lett., vol. 14, no. 6, pp. A93-A96, 2011.

[13] J. S. Foord, R. B. Jackman, and G. C. Allen, "An X-ray photoelectron spectroscopic investigation of the oxidation of manganese," Philos. Mag., vol. A49, pp. 657, 1984.

[14] T. Sato, S. Kasai, H. Okada, and H. Hasegawa, "Electrical properties of nanometer-sized schottky contacts on n-GaAs and n-InP formed by in situ electrochemical process," Jpn. J. of Appl. Phys., vol. 39-7B, pp. 4609, 2000.

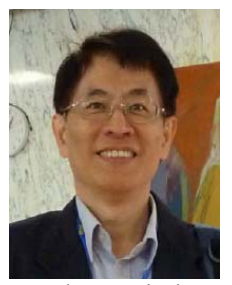

Yi Hu is the chairman in Department of Materials Engineering, Tatung University (TTU). Dr. Hu earned his BS and MS in Mechanical Engineering from TTU in Taiwan and his $\mathrm{PhD}$ degree in Materials Science and Engineering from the University of California, Los Angeles (UCLA) in USA. His research major is in the field of advanced ceramics and his $\mathrm{PhD}$ research involved developing novel organic-inorganic hybrid materials through sol-gel method. Dr. Hu has worked in the field of electronic ceramics, glass-ceramics, and sol-gel technique for more than 20 years and has published more than 100 peer-reviewed journal articles, symposium articles, and industry reports in this field. He was a member of the Materials Research Society-Taiwan (MRS-T) and of the The Chinese Ceramic Society (Taiwan).

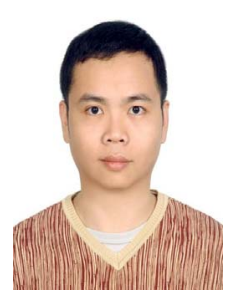

Jiun-Shing Liu was born in Tai-Chung, Taiwan. He received the BS degree in Materials Science and Engineering from the Feng Chia University (FCU) and MS in Materials Engineering from Tatung University (TTU) in Taiwan. He is currently a Ph. D. candidate Materials Engineering at TTU. His research interests include energy storage supercapacitor and electrochemical deposition technique, Sol-gel technique.

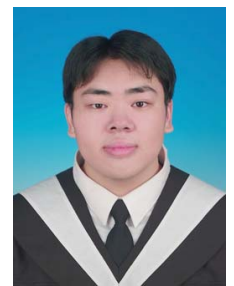

Tung-Cheng Liu was born in Taipei, Taiwan. He received the BS degree in Materials Science and Engineering from the Feng Chia University (FCU) and MS in Materials Engineering from Tatung University (TTU) in Taiwan. He is currently a Ph. D. candidate Materials Engineering at TTU. His research interests include solar cell technique, electrochemical deposition technique. 